\title{
Yielding, thixotropy and strain stiffening of aqueous carbon black suspensions
}

\author{
E. N'gouamba ${ }^{1}$, J. Goyon ${ }^{1}$, L. Tocquer ${ }^{1}$, T. Oerther ${ }^{2}$ and P. Coussot ${ }^{1}$ \\ ${ }^{1}$ Laboratoire Navier (ENPC-University Gustave Eiffel-CNRS), 77420 Champs sur Marne, France \\ ${ }^{2}$ Bruker BioSpin GmbH, Silberstreifen 4, 76287 Rheinstetten, Germany
}

\begin{abstract}
We study experimentally the rheological behavior of carbon black (CB) suspensions in water, at different ionic strengths and concentrations. We show by means of standard rheometry completed by local MRI-rheometry that these suspensions first appear to be thixotropic yield stress fluids: they exhibit a yield stress increasing with the time of rest, their apparent viscosity decreases under shear, and a viscosity bifurcation occurs around the yield stress, the fluid evolving either towards stoppage or to steady flow at a large shear rate for a small stress change. Then an original effect appears when we follow the mechanical state of the material in the solid regime by measuring its apparent elastic modulus at small deformation during a creep test under various stresses. In contrast with various other yield stress fluids for which the elastic modulus under small deformation appear to be constant for any deformation in the solid regime, for $C B$ suspensions this modulus widely increases while deformation increases up to yielding. We suggest that this strain stiffening effect finds its origin in the specificities of the (van der Waals) interactions and of the (rough) structure (aggregates) of the particles: the slight relative rotation of particles in contact due to deformation would on average tend to increase the net area of contact between particles, which stiffens the whole material structure. This is supported by the observation that the relative increase of elastic modulus is approximately proportional to sample deformation whatever the material characteristics (ionic force, concentration) and whatever the deformation history.
\end{abstract}

\section{Introduction}

Carbon Black (CB) particles dispersed in a liquid phase are used in a variety of applications such as reinforcing filler in polymer matrix [1], [2] stabilizers in polymer [3] or other materials [4] for protection against photo degradation, or as colour pigments in inks, paints and plastics [5]. CB is also used as a conductive filler to increase the conductivity of composite materials [6]. Finally, CB is used in the formulation of the electrolyte in redox flow batteries [7], [8] as it is considered to increase the conductivity through electronic percolation from the bulk to the collector.

Most rheological studies of $C B$ suspensions concern dispersions in oil. This indeed simplifies the mixture preparation as $C B$ is hydrophobic. This also simplifies the physical interpretation of data as it strongly limits the possible role of various effects such as evaporation, chemical reactions, electrostatic interactions between the particles, and adsorption of macromolecules onto the particles [9], [10] . In that case, coupling rheometry with ultrasonic measurements giving access to the deformation field, some studies focused on the solid-liquid transition under oscillations or creep [11] or on the fatigue dynamics under large amplitude oscillatory stress showing successive steps before full liquefaction [12] with smooth wall surfaces. Taking advantage of the conductivity of CB particles, Helal et al. [13] provided further information on the characteristics of the solid-liquid transition and its relationship with microstructure evolution (number of contacts, plastic events). Also, some organization of the structure in the direction of vorticity was shown for such materials at low shear rate in sufficiently confined geometries [14], [15], a phenomenon already observed in other systems such as laponite 
suspensions [16]. Finally, a universal stability diagram was established for this phenomenon for different types of colloidal systems (including CB suspensions), thus providing a general physical scheme [17].

More unexpected is the shear-thickening effect observed at high shear rates [9]. From microscopy observations the authors concluded that it should be due to an increase of the hydrodynamic volume of the particle aggregates after breakage, thanks to their fractal structures. More precisely, as in any colloidal gel, aggregation is promoted at low shear, and aggregates are broken at high shear rates. When aggregated, the fractal particles have the possibility to arrange into dense aggregates of interpenetrated particles, which have a density larger than the individual fractal particles. Suspensions of aggregates thus have a lower viscosity than the suspensions of fractal particles, in contrast with gels made of non-fractal particles. This approach was confirmed by further measurements [18]. Further descriptions of structure evolutions under shear or at rest were more recently provided [19-20].

These materials also exhibit a solid viscoelastic behavior below some critical stress [21-22], thanks to the formation of a loose colloidal gel, which might be expected to lead to thixotropic trends. This leads to some delayed catastrophic failure of the system beyond some critical stress [22]. However it appeared that the behavior of these suspensions is not thixotropic in the standard way: almost no evolution of the elastic modulus or yield stress was observed for increasing times of rest after flow stoppage [9], [18]. In contrast, the preshear may have a significant impact [10],[19]. This in particular led to show that it is possible to tune the material yield stress [18] to arbitrary low values by imposing progressively decreasing stress values while the fluid is flowing. On the other side it was shown that shearing history can tune concomitantly the elastic and the electric properties of these materials [13], which means that the material structure can be adjusted at will through an appropriate flow history.

From the environmental and economical point of view suspensions of $C B$ in water are more interesting [23] for electrolyte of redox flow batteries, than classical organic solvents due to the large amount of volumes involved. Mainly in view of this application, we here focus on the behavior of these suspensions in water, in contrast with most previous studies above described. We show that despite the hydrophobicity of carbon black (CB) particles, homogeneous aqueous suspensions of $C B$ can be prepared by dispersing gum arabic in the mixture. We first study these materials with conventional rheometry and MRI-rheometry (Magnetic Resonance Imaging) and show that, in contrast with suspensions of $C B$ in oil, suspensions of CB in water are basically "standard" thixotropic fluids. We then study their detailed rheological properties in the solid regime and find more original trends, including a strain stiffening effect. These observations provide further insight in the behavior of CB suspensions in general. We start by presenting the materials and procedures, and in particular the method of preparation of the water suspensions of $\mathrm{CB}$. Then we describe the data and finally we discuss the results.

\section{Materials and methods}

\section{II.1 Suspension preparation}

The suspensions consist of $\mathrm{CB}$ particles from Cabot (Vulcan XC72R) dispersed in deionized water with addition of various amounts of $\mathrm{CaCl}_{2}$ (Fisher Scientific). These particles result from the incomplete combustion of heavy hydrocarbons. The resulting small spherical elementary particles $(\approx 30 \mathrm{~nm})$ are associated by covalent links to form aggregates $(\approx 0.5 \mu \mathrm{m})$ which cannot be broken during flow [24], [25]. These aggregates may in turn be linked through van der Waals interactions, but these links may be broken during flow and reformed at rest. 
The samples are prepared as follows. Since CB particles are hydrophobic, gum arabic (from Fisher Scientific) is used as an emulsifier [26]. It is dissolved in water proportionally to the mass of $C B$ in order to (always) have a mass ratio of 0.2 between gum arabic and $C B$. The solution is left under magnetic stirring at least for 1 day, which ensures a complete dissolution of the polymer. $C B$ particles are then poured into the solution and thoroughly mixed with a paddle stirrer during $2 \mathrm{~min}$, followed by $15 \mathrm{~min}$ of sonication to break the reversible aggregates. After that, the blend is again thoroughly stirred with a paddle stirrer for $15 \mathrm{~min}$ and the desired amount of $\mathrm{CaCl}_{2}$ solution $1 \mathrm{M}$ is added 2 minutes before the end of stirring. The material is then sonicated for $5 \mathrm{~min}$. Those mechanical operations, finalized by a high preshear at $200 \mathrm{~s}^{-1}$ during $20 \mathrm{~min}$ in the rheometer before all rheological tests, allow us to get a homogeneous and reproducible material. Different volume fractions of $C B$ in water were tested. This volume fraction was deduced from mass fraction using the density of the elementary CB particles (i.e. $\rho=1.8$ g. $\mathrm{cm}^{3}[9]$ ).

\section{II.2 Particle interaction measurements}

CB particles are negatively charged particles in solution, due to the dissociation in water of the carboxylic and/or oxide function along their surface. The presence and proportion of these groups depend on the fabrication process [27-29]. By adding salt (here $\mathrm{CaCl}_{2}$ ) the surface charge of the particles decreases so that the electrostatic repulsion between particles decreases as well. A Zetasizer Nano (Malvern Instrument) was used to measure the $\zeta$-potential of CB particles in water. The $\zeta$ potential provides an estimate of the potential along the slipping plane between particles and interstitial fluid based on electrophoretic mobility [30]. Its value may be used to evaluate the dominant interaction type between particles by computing locally the electrostatic interactions (repulsive), then the total potential which is the sum of Van der Waals interaction (attractive) and electrostatic ones [31].

We carried out measurements of the $\zeta$-potential in highly dilute samples $(\approx 0.001 \mathrm{wt} . \%)$ for various ionic strengths, as it has been shown that the $\zeta$-potential in a concentrated suspension is close to the one measured in the dilute suspension [32]. The data (see Figure 1) show that the value of the $\zeta-$ potential remains low and does not depend much on the ionic strength: more precisely, it increases rapidly with ionic strength at low values then reaches a plateau for $I>88 \mathrm{mM}$. Estimating the interaction potential $W_{D L V O}$ from that between two parallel planes (justified by the non-sphericity of the particles) separated by a distance $D$ in an asymmetric electrolyte we get [30]:

$$
W_{D L V O}=-\frac{A}{12 \pi D^{2}}+2 \varepsilon \varepsilon_{0} \kappa \zeta^{2} \exp -\kappa D
$$

where $A=1.15 \times 10^{-19} \mathrm{~J}$ is the Hamaker constant of carbon in water, $\varepsilon_{0}=8.85 \times 10^{-12} \mathrm{~F} \cdot \mathrm{m}^{-1}$ the vacuum permittivity and $\varepsilon=78.4$ the relative permittivity of the medium, $\zeta$ the $\zeta$-potential value, and $\kappa^{-1}$ the Debye length, which here expresses as $\kappa^{-1}=\left(\varepsilon_{0} \varepsilon k_{B} T / 2 e^{2} N_{A} I\right)^{1 / 2}$, in which $k_{B}=$ $1.38 \times 10^{-23} \mathrm{~J} . \mathrm{K}^{-1}$ is the Boltzmann constant, $\mathrm{T}=298 \mathrm{~K}$ the temperature, $e=1.602 \times 10^{-19} \mathrm{C}, N_{A}=$ $6.022 \times 10^{23} \mathrm{~mol}^{-1}$ the Avogadro number and $I$ the ionic strength which here may be written as $I=$ $3\left[\mathrm{CaCl}_{2}\right]$ in mol. $\mathrm{L}^{-1}$.

Using the values measured for $\zeta(<-20 \mathrm{mV})$ we deduce that, in our range of ionic strengths, the second (electrostatic) term in the right hand-side of equation (1) is negligible with regards to the first term for our range of materials and at any distance, so that the total potential is systematically attractive and independent of the ionic strength (see Appendix 1). This implies that repulsive forces remain negligible and the particles finally attract each other until they are in contact. However, it is likely that due to the 
particles roughness, in particular resulting from their specific aggregate structure (see above), these contacts take place along a few regions of very limited areas ("contact points"), which explains that the attractive force is limited and such links may be broken easily if the material is forced to flow. Finally, in the following we study materials by varying the parameters (ionic strength, concentration) so as to still obtain measurable yield stresses which can be associated with the formation of a structure, meaning that the interactions between particles play a major role on the rheological behavior. This allows to test different conditions to check the generality of our findings.

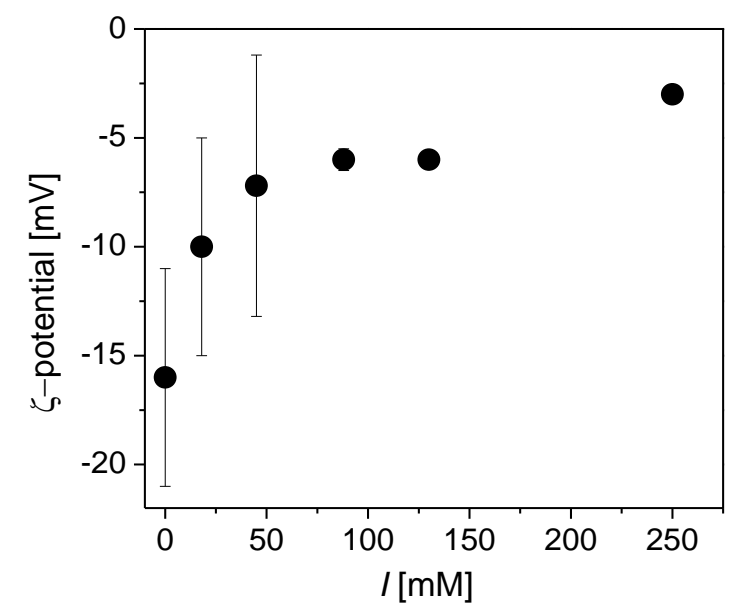

Figure 1. $\zeta$-potential as a function of ionic strength for a dilute aqueous $C B$ suspension.

Let us now examine the role of GA (gum arabic) in this context. Although GA is often used as an emulsifier this is not the role we expect for it here. Indeed, the typical GA molecule size is $\approx 20 \mathrm{~nm}$ [33], [34], and usual emulsification with GA is obtained thanks to the adsorption of these molecules onto the surface of droplets which exhibit a much larger size (typically of the order of 1 micron) [35]. The layer formed by these molecules prevents the droplets from coalescence. In our case, the GA molecule size is of the same order as the $C B$ elementary particle size $(30 \mathrm{~nm})$ and no coalescence can occur. The GA molecules are dispersed in water and considering their size (and the mass fraction with regards to $\mathrm{CB})$, their apparent volume fraction is of the order of $64 \%$ for example for a $\mathrm{CB}$ concentration of $10 \%$ (and taking a typical molecular mass of $3 \times 10^{5} \mathrm{~g} / \mathrm{mol}$ ). That means that they occupy the whole volume of liquid and are close to each other, and they are situated everywhere around the CB particles. Under these conditions, we believe that they essentially tend to bring water (the water contained in the pellet) in contact with the CB particle surface, and thus favor a good wetting of their surface. By the way, there might be some adsorption of these molecules to the CB particles but it is likely that when mixing the suspension the major effects will be the colloidal interactions between the $C B$ particles, and more precisely their attractive forces leading to direct contacts while GA molecules will be situated around these points of contact. As explained below, our rheological results support the assumption that these molecules do not play a significant role in the mechanical behavior here described.

\section{II.3 Rheometry}

The rheological tests are performed at room temperature $\left(25^{\circ} \mathrm{C}\right)$, with a stress-controlled-rheometer (Malvern Kinexus), equipped with a concentric cylinder geometry. The wall surfaces are serrated to avoid slippage, the gap size is $e=1.25 \mathrm{~mm}$ and the height of the inner cylinder is $H=37.5 \mathrm{~mm}$. This 
gap a priori allows to avoid the formation of vorticity-aligned flocs which have been observed in several dispersions of attractive particles when sheared in confined geometry [14]-[17]. The independence of the rheological measurements on the geometry has been checked through tests with parallel disks geometries (data not shown).

The behaviour of $\mathrm{CB}$ suspensions may a priori be sensitive to their flow history. Therefore, a preshear (200 s $\mathrm{s}^{-1}$ during $60 \mathrm{~s}$ ), is applied prior to any measurement, in order to erase the flow history, which sets up the sample in a reference state. Then the material is left at rest during a certain amount of time, which allows the structure of the material to rebuild from this reference state. We checked that this procedure ensures a good reproducibility of the initial state of the suspensions. Various rheometrical procedures have then been used which are described in the Experimental Section.

\section{II.4 MRI velocimetry}

MRI (Magnetic Resonance Imaging) velocimetry measurements in Couette cell have been carried out in a Bruker Biospin GmbH. The NMR method for the velocity measurement is a "Pulsed Gradient Spin Echo Velocity Imaging" sequence [35], generating 2D spatially resolved velocity maps from which 1D velocity profiles (orthoradial velocity $\left(v_{\theta}\right)$ ) across the gap of the Couette cell are extracted. The Couette cell characteristics is the following: the inner cylinder, made of polyether ether ketone (PEEK), has a radius of $7 \mathrm{~mm}$ and a height in contact with material $(H)$ of $60 \mathrm{~mm}$, the gap size is $e=2 \mathrm{~mm}$. The inner cylinder is mounted to a motor, the rotation velocity was varied in the range 10 to $600 \mathrm{rpm}$ with a radial resolution of $55 \mu \mathrm{m}$. The outer cup is kept static. The Couette cell is immerged into a static field of $300 \mathrm{MHz}(7 \mathrm{~T})$. In the plane perpendicular to the axis of the cylinder, the acquisition window is a parallelogram of radial length $7 \mathrm{~mm}$ and tangential width $18 \mathrm{~mm}$.

1D velocity profiles provide a qualitative information on the flow characteristics, but they may be used in a quantitative way to determine the constitutive equation of the material. Indeed, it can be demonstrated from momentum equation that in the flowing region the stress at a distance $r$ from the central axis expresses as $\tau=T / 2 \pi H r^{2}$, in which $T$ is the torque applied on the inner cylinder. This implies that the stress varies by a factor 1.65 from the inner to the outer cylinder, and for a yield stress fluid the shear rate may increase by several orders of magnitude in such a range of stresses. We thus expect a significant gradient of shear rate along the gap of the Couette cell. This may be used to get detailed information on the constitutive equation of the material. Indeed, the local shear rate at a distance $r$ writes $\dot{\gamma}=v_{\theta} / r-d v_{\theta} / d r$. Then we can plot in a rheogram the local shear rate associated with the local shear stress for different distances $r$ to get a series of stress vs shear rate data corresponding to the local, effective, rheological behaviour of the material [37]. Note that, under these conditions we do not have a single shear rate associated with each velocity profile but a more or less wide range of shear rates. This range of shear rates appears in Figure 5 , for each rotation velocity.

\section{Experimental results}

\section{III.1 Sweep tests}

The rheological behaviour of the material is first observed from the simplest test, i.e. a sweep test. This consists to impose a logarithmically increasing ramp of stress from 1 to 100 Pa over $180 \mathrm{~s}$, then a decreasing stress ramp over the same range (see Figure 2). The stress vs shear rate curve observed during the increasing step exhibits a shape typical of a yield stress fluid: a rapid stress increase below a critical stress while the shear rate remains around a low value, then a rapid increase of the shear rate 
for a slight increase of the stress beyond a critical value. Actually, the first stage corresponds to deformations in the solid regime and the apparent shear rate does not correspond to a steady flow of the material in the liquid regime but to the successive steps of deformation increase as the stress is increased in time. The stress beyond which the rapid increase of shear rate is observed is the so-called "static yield stress" $\left(\tau_{c, s}\right)$. Note that this value of $\tau_{c, s}$ is not either a simple rheological property of the fluid as it can depend on the rate of increase of the stress along the ramp and on the time of rest between the end of preshear and the beginning of the sweep test.

The suspension presents an important hysteretic response, i.e. the stress vs shear rate curve in the decreasing stress ramp, is situated significantly below the increasing stress ramp. This hysteretic response, well known as 'thixotropic loop', clearly shows the dependence of the sample on its shear history and thus evidences its thixotropic behaviour. The stress for which the flow stops during the decreasing stress ramp is called the "dynamic yield stress" $\left(\tau_{c, d}\right)$ (see Figure 2 ). It follows that, due to this thixotropic behaviour, the results of such a test can hardly be used to extract the steady state flow characteristics of the material, since any data point may correspond to a transient state. More complete experiments are necessary to extract the detailed behaviour in the solid and the liquid regimes.

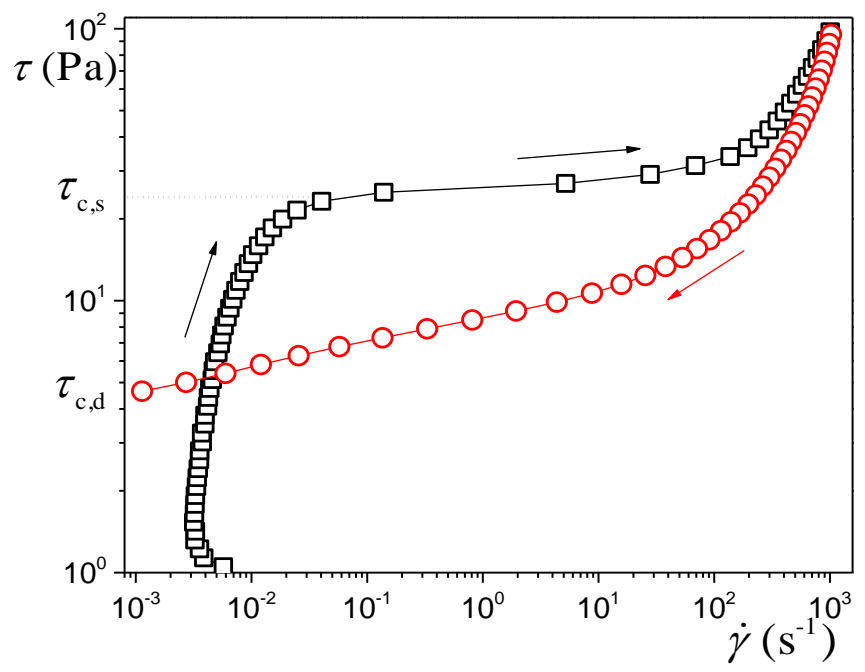

Figure 2. Sweep test (see text) for an aqueous CB suspension $\Phi_{v}=7.5 \%$ and $I=88 \mathrm{mM}$ and resting time of $60 \mathrm{~s}$ : increasing (squares) then decreasing (circles) ramp. The values $\tau_{c, s}$ and $\tau_{c, d}$ are the apparent static and dynamic yield stress under these specific conditions.

\section{III.3 Creep tests}

We now observe in more details the solid-liquid transition from a series of creep tests under various stress values, each time starting from the same state of the material (preshear + time at rest (mentioned in captions)). For an imposed stress, we follow the strain vs time over $30 \mathrm{~s}$, then stop the test, preshear the sample and repeat the same procedure for another stress level.

As usually observed for yield stress fluids [38] two distinct regimes clearly appear: below a critical stress (here $11 \mathrm{~Pa}$ ) the strain tends to a plateau so that its value appears to be limited, i.e. it remains below a critical value $\gamma_{c}$; beyond this critical stress the strain continuously increases and finally reaches a 
regime where it increases linearly in time (slope 1 in logarithmic scale), this corresponds to a flow at a constant shear rate. The former regime can be considered as the "solid regime", the latter as the "liquid regime". This critical stress is the true static yield stress $\tau_{c, s}$ of the suspension for a given flow history (preshear + rest). The value of $\tau_{c, s}$ as considered in the following is determined according to this procedure.

As for other thixotropic materials [39], it is remarkable that this material is unable to flow in steady state at a shear rate below a finite critical value. Indeed, when the stress is decreased towards the yield stress, the shear rate remains larger than about $9 \mathrm{~s}^{-1}$, and finally when the applied stress is just below the yield stress the material stops flowing (solid regime) (see Figure 3 ). Thus the CB suspension presents a restricted range of $\dot{\gamma}$ in its stationary regime and an abrupt transition from the solid regime $(\dot{\gamma}=0)$ to the liquid regime $\left(\dot{\gamma} \geq 9 s^{-1}\right)$. The solid-liquid transition is finally marked by a "collapse" or an "avalanche" which appears for a critical stress and a critical deformation $\gamma_{c} \approx 120 \%$ (see Figure 3).

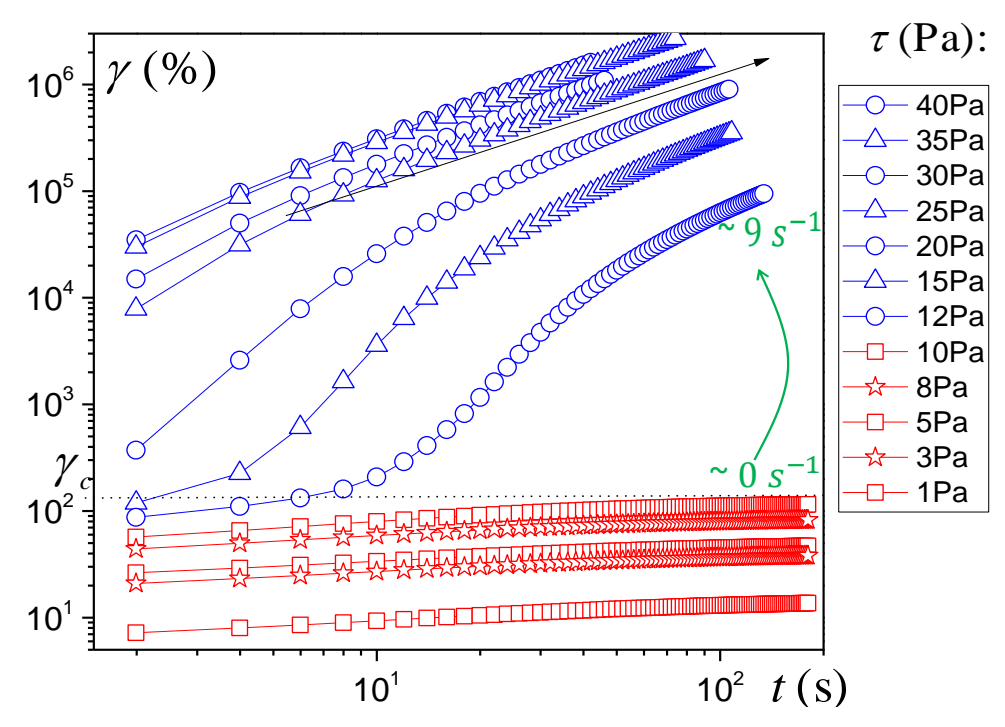

Figure 3. Creep tests for an aqueous CB suspension $\Phi_{v}=7.5 \%$ and $I=88 \mathrm{mM}$ after a resting time of $60 \mathrm{~s}$ : shear strain vs time for different stress values. For a stress (squares and stars) smaller than a critical value (say, $11 \mathrm{~Pa}$ ) the curves tend to a plateau and a shear rate around $0 \mathrm{~s}^{-1}$. For a larger stress (circles and triangles) the curves tend to a slope 1 indicating a steady state flow, at a shear rate larger than about $9 \mathrm{~s}^{-1}$.

\section{III.3 Local rheometry (MRI)}

Since flow of thixotropic materials may easily be unstable or develop heterogeneities [38] it is useful to check the consistency of the above conclusions with internal observations, i.e. by measuring velocity profiles thanks to Magnetic Resonance Velocimetry. The material is first presheared by imposing a high rotation velocity, i.e. $600 \mathrm{rpm}$, to the inner cylinder during two minutes, so that the material is a priori fully flowing in steady state in the gap. Then we decrease the rotational velocity to successively lower values (steps) and, at each step, we wait until obtaining a stationary velocity profile. 


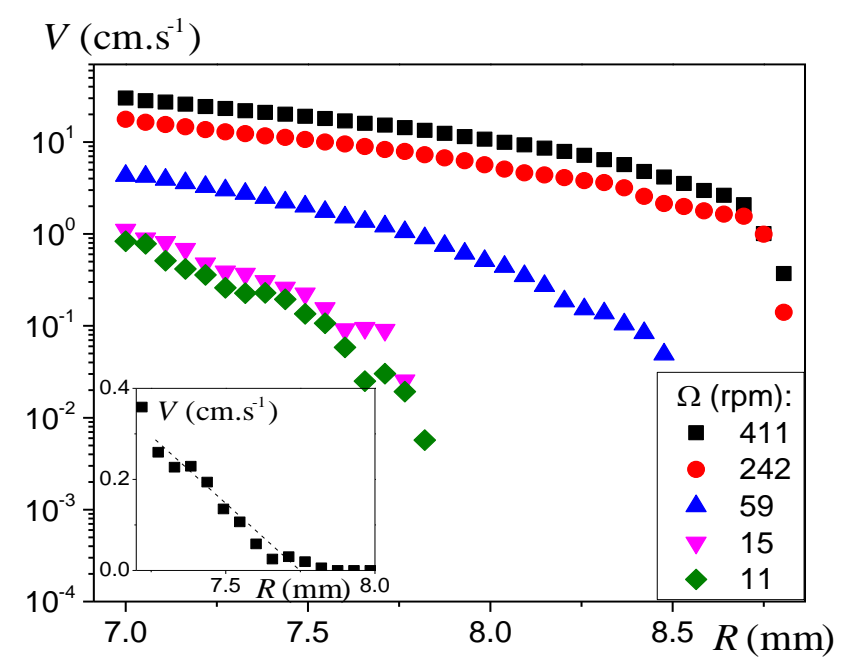

Figure 4. Velocity profiles for steady states flows of the aqueous CB suspension $\Phi_{v}=$ $7.5 \%$ and $I=88 \mathrm{mM}$ in the Couette cell as determined by MRI for different rotation velocities. The inset shows a zoom of the velocity profile for a rotation velocity of $11 \mathrm{rpm}$, around the liquid-solid transition: the slope of the velocity profile (and thus the shear rate) remains approximately constant at the approach of the transition.

For each rotational velocity the velocity profile was measured every minute over five minutes. In each case a stationary profile was observed after one minute. This profile is presented in Figure 4 for the different rotation velocities. At low rotational velocities, unsheared regions, i.e. in which the velocity is equal to zero, appear (see Figure 4). Moreover, the transition between the sheared and the unsheared region is abrupt, i.e. the slope of the velocity profile, and thus also the shear rate, remains constant at a significant distance from the unsheared region (see inset of Figure 4). The corresponding shear rate $\left(\dot{\gamma}_{c} \approx 7 \mathrm{~s}^{-1}\right)$ is close to the estimated critical one associated with the solid-liquid transition (see Figure 3). Consistently with the above description of the characteristics of the solid-liquid transition, and as already shown for various materials [37], [40] the flow curve of the material deduced from these local measurements takes the form of a curve starting at a shear rate $\dot{\gamma}_{c}$, i.e. no steady state flow can be observed at a shear rate lower than $\dot{\gamma}_{c}$, then the stress increases as the shear rate is increased beyond $\dot{\gamma}_{c}$ (see Figure 5). Since it is not possible to get a homogeneous steady state flow below $\dot{\gamma}_{c}$, when a lower shear rate is imposed shear-banding appears [41]. This effect also likely occurs during a sweep test: during the increasing stress ramp the shear is localized in a relatively thin layer whose thickness would rapidly grow, which would lead to a rapid increase of the shear rate; during the decreasing stress ramp, the material is initially fully sheared and liquefied, so that we get the effective flow curve for shear rate larger than $\dot{\gamma}_{c}$; for lower shear rate shear-banding in principle occurs. At last note that in a Couette geometry, the shear-banding is associated with a sheared layer thickness decreasing to zero as the stress is decreased to the yield stress. It is likely that the flow in this thin layer leads to a slight depletion of the solid concentration which allows a flow under stress smaller than the yield stress, which would explain the decreasing stress ramp curve situated at stresses significantly smaller than the effective yield stress for shear rate lower than $\dot{\gamma}_{c}$ (see Figure 5). 


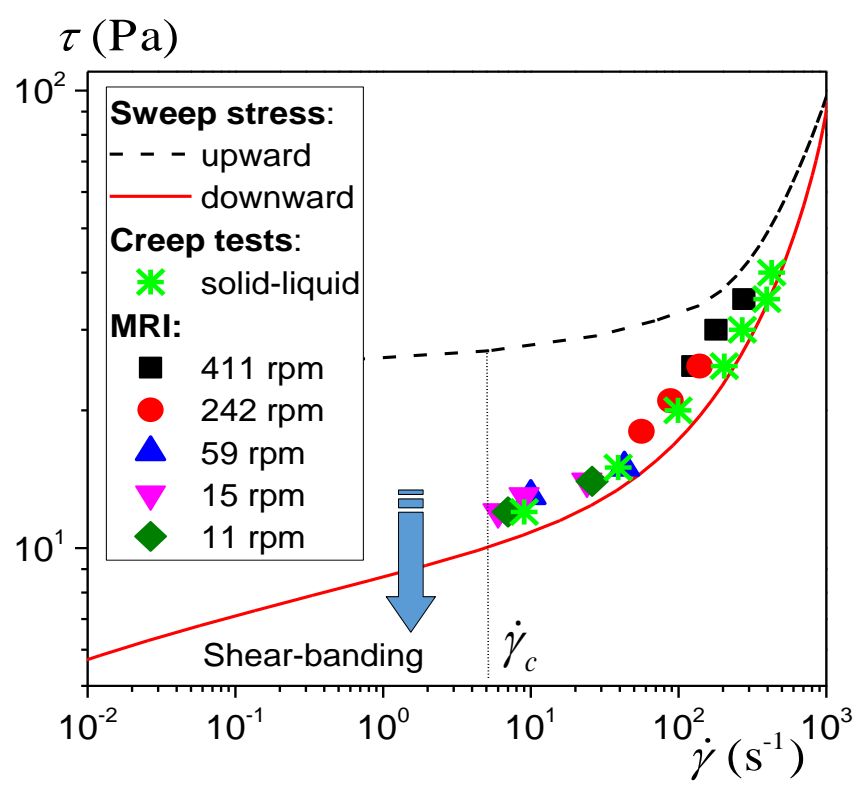

Figure 5. Flow curves for the aqueous $\mathrm{CB}$ suspension $\Phi_{v}=7.5 \%$ and $I=88 \mathrm{mM}$ obtained in different ways: sweep test, creep tests, MRI velocimetry at different rotation velocities.

The thixotropic properties of this material are confirmed by the observation of the evolution of the static yield stress and the elastic modulus (in the linear regime, see next Section) as a function of the time at rest (see Figure 6). Strong thixotropy effects are observed: the elastic modulus increases by a factor larger than 10 over one hour, which shows that the structure of the material significantly reinforces at rest. We have no physical explanation for this phenomenon but we can remark that such a variation is very similar to that observed for lime or cement pastes [42-43], whose elastic modulus increase was considered to find its origin in the evolution of direct contacts between particles. The yield stress also increases but more slowly, which in particular illustrates the fact that the yield stress and the elastic modulus are not simply correlated as already observed in some complex systems such as cement paste [43] or waxy suspensions. For example the yield stress is not simply equal to the elastic modulus times a critical deformation, because yielding occurs for a deformation much larger than that for which the linear regime is observed [44]. 


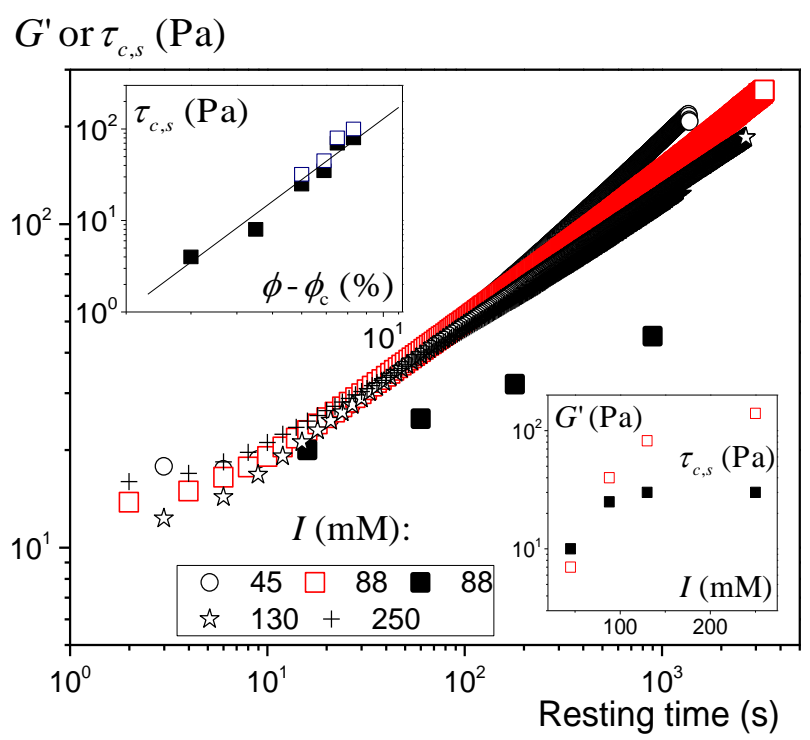

Figure 6. Elastic modulus (open symbols) and static yield stress (filled symbols) of aqueous $C B$ suspensions $\left(\Phi_{\mathrm{v}}=8.5 \%\right)$ as a function of the resting time for different ionic forces. The elastic modulus has been rescaled by the elastic modulus value for $\mathrm{I}=88 \mathrm{mM}$ after $60 \mathrm{~s}$ at rest, i.e. we represent $G_{I}^{\prime}(t) G_{I=88 m M}^{\prime}(t=60 \mathrm{~s}) / G_{I}^{\prime}(t=60 \mathrm{~s})$. The top left inset shows the yield stress as a function of the solid fraction minus the critical one (here $\phi_{c}=2.5 \%$ ) for $I=88 \mathrm{mM}$ and $60 \mathrm{~s}$ of rest (filled squares) and $180 \mathrm{~s}$ of rest (open squares). The bottom right inset shows the elastic modulus (open squares) and the yield stress (filled squares) for $\phi=8.5 \%$ after $60 \mathrm{~s}$ at rest as a function of the ionic force.

\section{III.4 Impact of suspension composition}

As expected the yield stress of the suspension increases with the particle concentration (see top left inset of Figure 6). The density of the network is increased, which implies a larger density of interactions. The power-law variation (with an exponent $3 \pm 0.5$, depending on the fitting way) of the yield stress as a function of the distance from the critical solid fraction $\left(\phi_{c}\right)$ for which no yield stress is observed, suggests some kind of percolation phenomenon. Note that a similar exponent (i.e. 3.4) was found for the yield stress of $C B$ suspensions in mineral oils [45], and the fractal nature of the structure was considered to explain this rather large value.

An important feature is that the ionic strength does not affect the aging characteristics of the suspensions: the rescaled $G^{\prime}$ curves evolve in a similar way. A similar result was recently obtained with silica suspensions in water with salt. In that case it was demonstrated that the elastic modulus finds it origin in direct contacts between particles which evolve in time, rather than in an increase of the interaction number thanks to geometrical rearrangements [46]. This suggests a similar origin of the strength of the $\mathrm{CB}$ suspensions.

However, we also observe that the yield stress and the elastic modulus increase with the ionic strength for $K 88 \mathrm{mM}$ before apparently tending to a plateau for higher I (see bottom right inset of Figure 6). This is unexpected since we have seen that the attractive van der Waals force is a priori dominant. A possible explanation is that the electrostatic force would play a role in the very first times after preparation or preshear, when the particles reach their final spatial distribution: lower electrostatic force could lead to a lager density of interparticle contacts. 


\section{III.5 Oscillation tests}

In order to further appreciate the characteristics of the solid-liquid transition and observe the possible existence of a linear domain we performed a standard ramp of oscillating deformation (at a frequency of $1 \mathrm{~Hz}, 5 \mathrm{~s}$ per data point) of increasing amplitude on the aqueous carbon black suspensions. Note that since we worked with a stress-controlled rheometer it is preferable to impose the stress amplitude rather than the strain amplitude to avoid feedback noise, but we finally represent the data as a function of the resulting strain amplitude. We effectively observe a linear domain, typically up to about $\gamma=5 \%$ , where the elastic modulus seems to be independent of the strain amplitude (see Figure 7). We can remark a very slight increase of $G^{\prime}$ in this regime, which might be due to some slight rearrangement or aging of the structure independent of the deformation. We will not discuss further this minor effect as compared to that occurring at larger strain. Indeed, for larger strain amplitude, $G^{\prime}$ slowly increases up to a maximum and then collapses towards low values and the cross-over with the $G^{\prime \prime}$. This significantly contrasts with the trend observed generally for simple or significantly thixotropic yield stress fluids (see Figure 7): for strain amplitude beyond the linear domain, $G^{\prime}$ progressively decreases and finally rapidly decreases towards much lower values; this is generally associated with a progressive breakage of the structure and appearance of plastic events up to the full breakage [47]. For water suspensions of $C B$ we thus have an original effect in which the structure seems to be strengthened by large deformations before breakage. Note that this particular behaviour appears to be more striking at low ionic strengths and tends to vanish for the highest ones (see Appendix 2). This increase of $G^{\prime}$ with the strain is reminiscent of the strain stiffening observed with hydrogels [48] [49] or colloidal gels [50][51]. However, with this procedure we simultaneously impose larger deformations and measure the material properties associated with the same deformation. In order to better describe and understand these effects we have studied this phenomenon in different ways consisting to impose deformation and measure the material properties from additional probing.

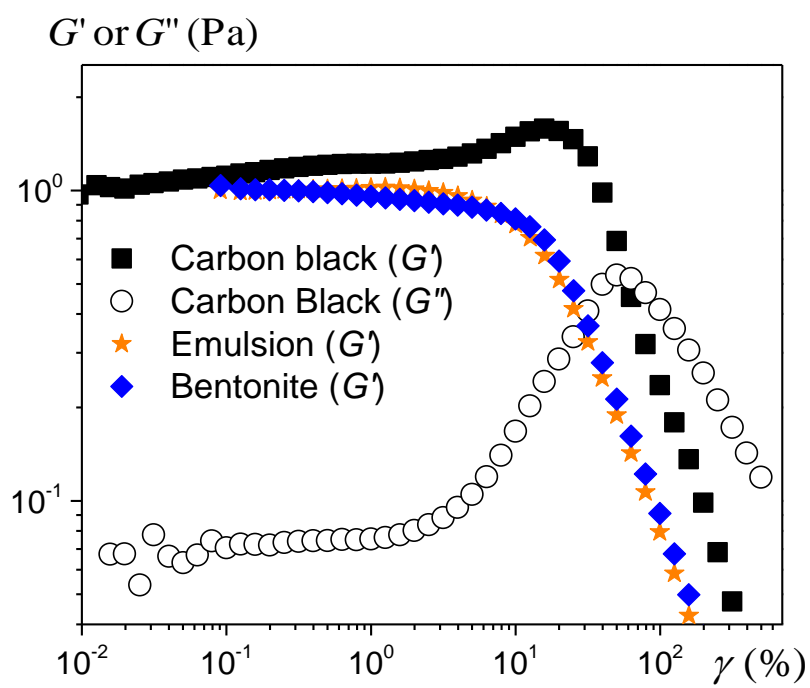

Figure 7. Elastic and viscous moduli for an aqueous $\mathrm{CB}$ suspension $\left(\Phi_{v}=8.5 \%\right.$ and $I=$ $45 \mathrm{mM}$ ) during an increasing ramp of strain amplitude. Elastic modulus under similar measurement conditions for a bentonite and an oil in water emulsion. In order to strictly compare the different evolutions as the function of the strain amplitude, the moduli for each material have been rescaled by their value at low deformation (50 Pa for CB, $360 \mathrm{~Pa}$ for the emulsion, $500 \mathrm{~Pa}$ for the bentonite). 


\section{III.6 Recovery tests}

We performed creep and recovery tests in the solid regime of the $\mathrm{CB}$ suspensions. As usual, the sample is first presheared then left at rest during $60 \mathrm{~s}$, then a constant stress $\tau<\tau_{c, s}$ is applied during a short time (creep test), which leads to some deformation. The stress is then released and we follow the deformation in time, which tends to a plateau (see inset of Figure 8). The difference between the initial deformation at the end of the creep test and the plateau value, $\gamma_{E}$, corresponds to the elastic component of the deformation for the applied stress in the creep test. In Figure 8 we plot the value of this elastic component as a function of the applied stress in the creep test. We observe that the deformation increases (with the stress) more slowly as the stress is increased, leading to a convex stress vs strain curve. These results remarkably differ from those obtained with various other simple or thixotropic yield stress fluids, for which the elastic component of the deformation measured in a similar way, increases more rapidly as the stress is increased, leading to a concave stress vs strain curve. We illustrate this difference by a comparison of the results for CB with those for a Carbopol gel which are representative of the results for the above mentioned systems. It thus appears that here, unexpectedly, the material tends to be more rigid as it is more deformed in its solid state. Indeed, similarly with the increase of $G^{\prime}$ with strain, we here observe an increase of the apparent shear modulus (stress to strain ratio) of the material with strain, which may be described as a strain stiffening.

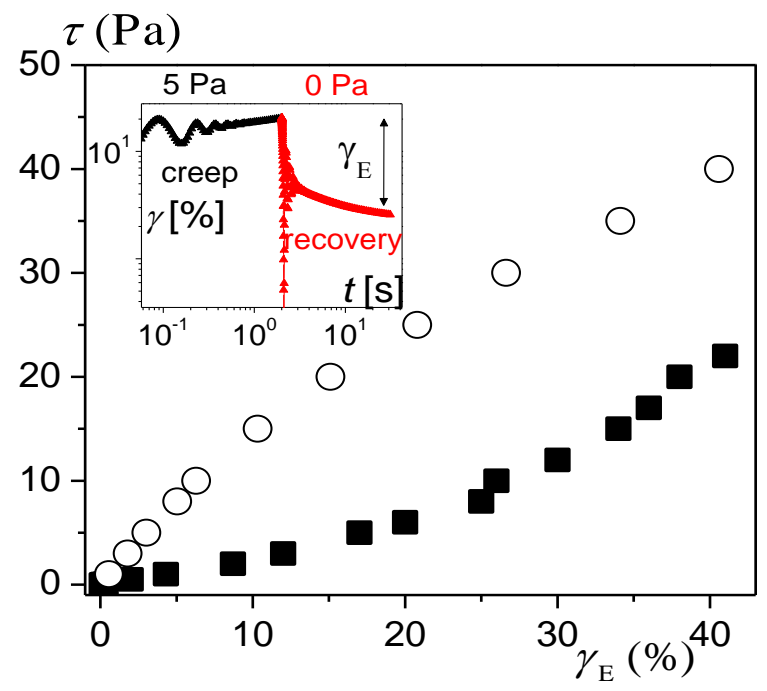

Figure 8. Shear stress vs elastic deformation deduced from creep then recovery tests for aqueous CB suspension $\left(\Phi_{v}=8.5 \%\right.$ and $\left.I=88 \mathrm{mM}\right)$ (squares). The open circles correspond to data for Carbopol from N'gouamba et al. [52] obtained under similar experimental conditions. The inset shows an example of creep then recovery test for the CB suspension for $\tau=5 \mathrm{~Pa}$ showing residual and elastic deformations.

\section{III.7 Superimposition tests}

In order to probe the structure of the material during creep tests in the solid regime, we used a quite original and robust test [53] which consists in superimposing to a constant stress $\left(\tau_{0}\right)$, a stress oscillation of small amplitude $\varepsilon$, so that the total stress writes $\tau=\tau_{0}+\varepsilon \sin \omega t$, with $\omega$ the frequency of the oscillations. The frequency is kept constant at $1 \mathrm{~Hz}$, and results are generally independent of the 
frequency for such materials [54]. The resulting deformation can be well represented as $\gamma=\gamma_{0}+\eta \sin (\omega t-\varphi)$, in which $\gamma_{0}$ and $\eta$ are deformation amplitudes and $\varphi$ the phase shift. The amplitude of the oscillations $(\varepsilon)$ is chosen to induce an additional deformation in the linear regime of the materials. The typical value resulting from this choice for $\varepsilon$ was $10^{-3}$. An elastic modulus $\left(G^{\prime}\right)$ associated with the material behaviour under such oscillations is then computed as $G^{\prime}=(\varepsilon \cos \varphi) / \eta$.

Typical results are here shown for the suspension at $I=88 \mathrm{mM}$ for various concentrations of particles. We carry out creep tests at different stress values, always starting from the same initial material preparation (here preshear then rest for $60 \mathrm{~s}$ ). We thus get the corresponding creep test curves (i.e. deformation vs time) for each stress level. As already noticed, the resulting deformation varies in a wide range (up to a value of the order of 100\%) when the stress is increased up to the yield stress (see Figure 9a). In addition, we may remark that a slight creep flow is observed under these conditions, but this will not affect the following analysis.

During these creep tests we superimpose small stress oscillations providing an apparent $G^{\prime}$ value (see definition above). It appears that, except during the first few seconds, this parameter increases in time quite similarly to the elastic modulus measured at rest (no applied stress) (see Figure 9b), i.e. in a logarithmic scale the different curves $G_{\tau}^{\prime}(t)$ are parallel to the $G_{\tau=0}^{\prime}(t)$ curve at rest. The major point here is that although they evolve similarly, their level is significantly larger for larger applied stress during these creep tests (see Figure 9b). In order to further quantify this trend we can determine the factor by which $G^{\prime}$ is increased (neglecting the initial fast increase at the beginning of the creep test) with regards to the situation of zero stress, as a function of the applied stress. This factor is defined as $a(\tau)=G_{\tau}^{\prime}(t) / G_{\tau=0}^{\prime}(t)$. In Figure 10 we plot the values of $a(\tau)$ (times $G_{\tau=0}^{\prime}(180 s)(\mathrm{Pa})$ ) as a function of the applied stress for different concentrations. It appears that the elastic modulus, which is larger for higher particle concentration, increases in the same way as a function of the distance from the yield stress (see Figure 10): the curves obtained for $a(\tau)$ approximately follow a similar curve shifted vertically when represented as a function of the ratio of the stress to the yield stress.
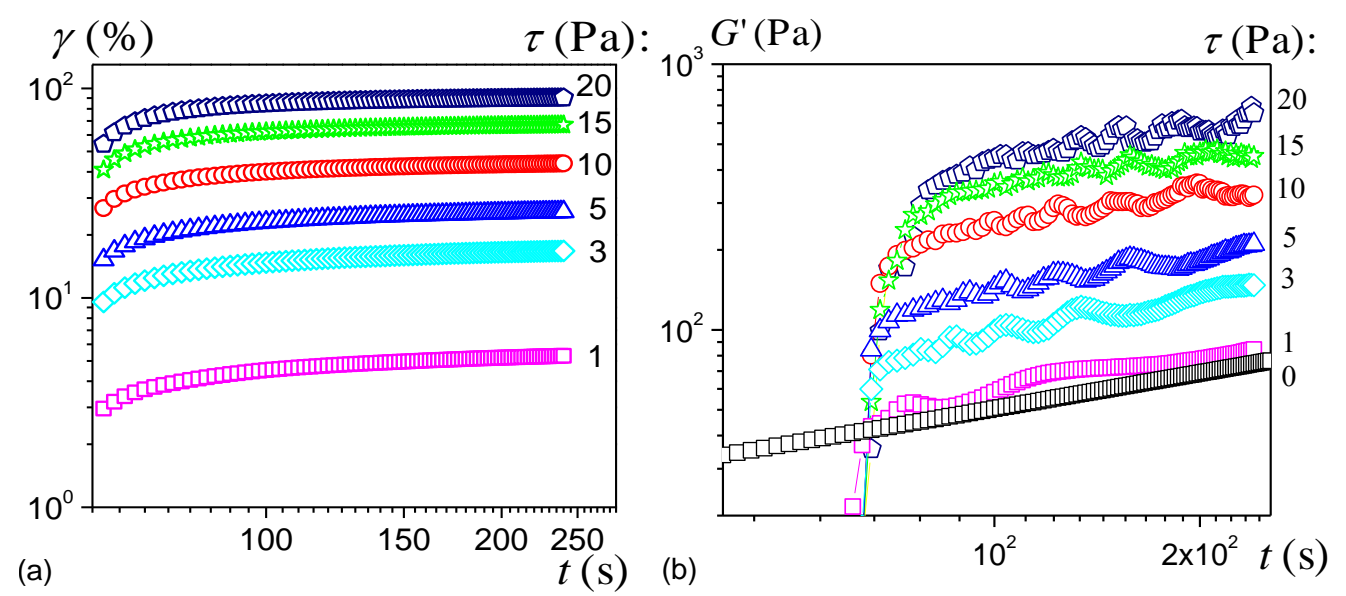

Figure 9. (a) Strain vs time during creep tests at different stress values for an aqueous $C B$ suspension $\left(\Phi_{v}=8.5 \%\right.$ and $\left.I=88 \mathrm{mM}\right)$; (b) Elastic modulus vs time during these creep tests, as measured under very small superimposed stress amplitude. 
An important point to note is that the preshear then rest for $60 \mathrm{~s}$ allows to erase this effect in the sense that the elastic modulus measured after this process takes the same value as at the beginning of the test, i.e. it does not keep the value it has reached at the end of one of the creep test. However, the phenomenon is reversible: if, after this rejuvenation, we start again imposing a creep test we find the same strain stiffening effect (see Appendix 3).

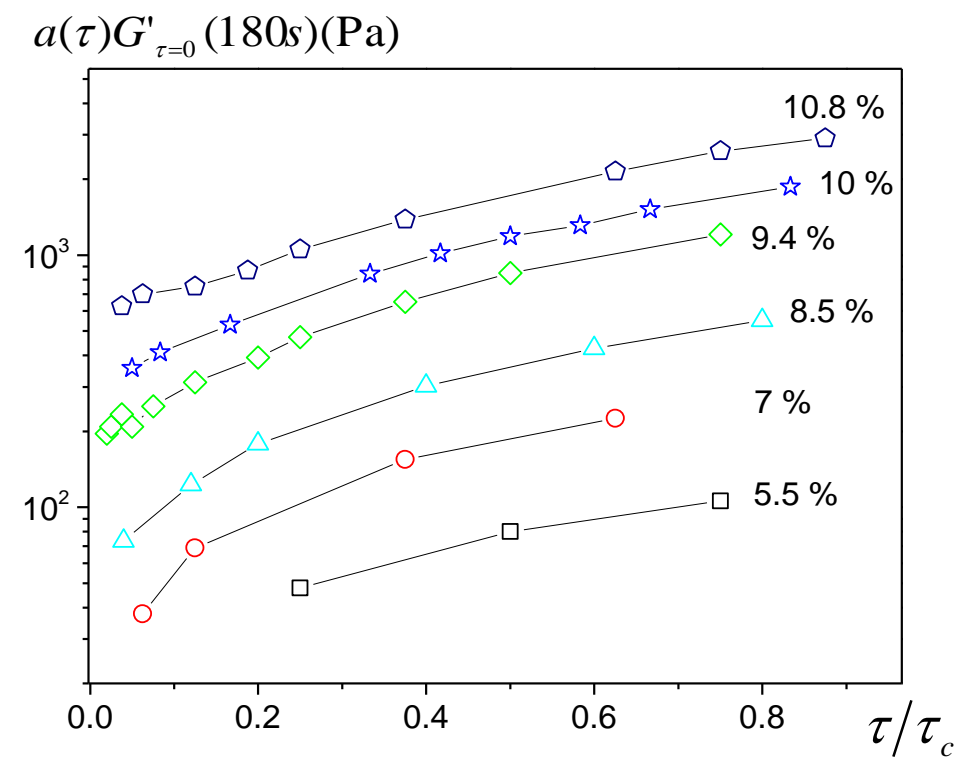

Figure 10. Initial elastic modulus times coefficient $a$ (see text) extracted from elastic modulus measurement under small oscillations during creep tests at different stress values for water CB suspensions at different concentrations. See values of yield stress and $G_{\tau=0}^{\prime}(180 \mathrm{~s})$ as a function of concentration in Appendix 4.

In order to test the possible evolutions of other properties of the material during such a superimposition test we now impose an increasing stress ramp just at the end of each of the above creep tests, and follow the apparent shear rate in time. It is remarkable that the flow curve obtained under such conditions is negligibly affected by the material properties at the end of the creep test: the flow curves fluctuate around a mean value without specific trend (see Figure 11). Thus, although the apparent elastic modulus under small oscillations can significantly increase (typically up to a factor 10), the apparent static yield stress remains approximately constant in any case (see Figure 11). This confirms that the physical origin of the yield stress differs from that of the elastic modulus. In particular we can suggest that the yield stress value is mainly associated to some kind of disentanglement of the particles, which involves a significant dissipation of energy, and that the initial breakage of the elastic structure plays a minor role in this process. 


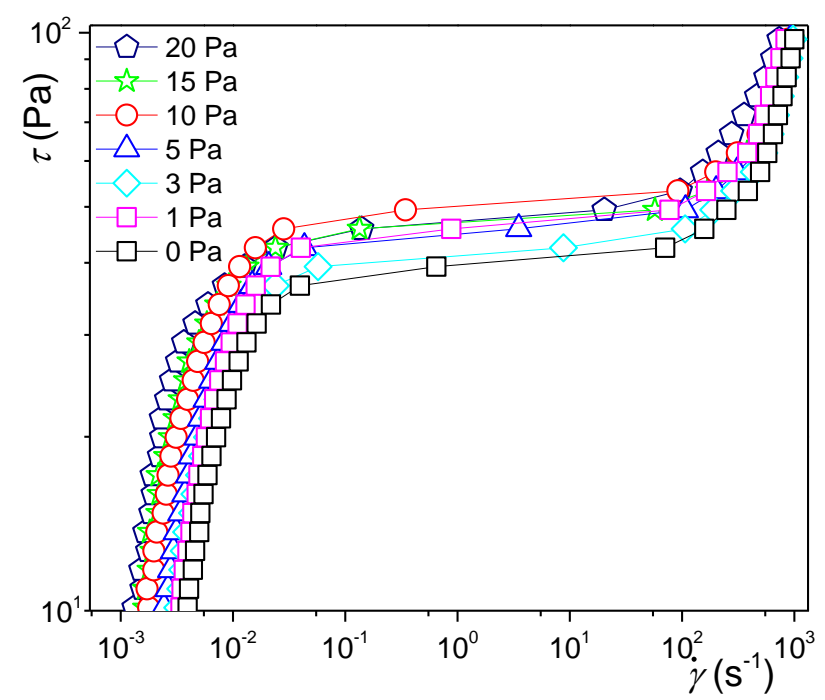

Figure 11. Apparent flow curve from increasing stress ramp for material of Figure 9 after each creep test plus oscillation as described in Figure 9.

\section{Discussion}

We have seen that, in contrast with suspensions of CB in oil [18], water suspensions of CB essentially behave as thixotropic yield stress fluids. They exhibit a static yield stress which increases with the resting time. When a stress is applied to the suspension at rest, the solid-liquid transition occurs rather abruptly for a stress larger than the critical stress, leading to a rapid increase of the shear rate. Consistently with such an observation, the flow curve of the material is truncated, i.e. no steady state flow can be obtained below a critical shear rate. So far this type of rheological behaviour has been typically observed with aggregated suspensions of colloidal particles such as clay or silica suspensions [40], and was described as resulting from the relatively long relaxation of the structure which needs some time to restore after breakage [55]. As a consequence the structure progressively reforms at rest, which explains that the apparent yield stress or the elastic modulus increase with the time at rest. Such a structure evolving in time might result from progressive collective rearrangements between particles thanks to attractive interactions [56]. It might also result from the progressive strengthening of contact interactions of the particles with negligible spatial rearrangements, as discovered recently [46]. The strong and continuous increase of the elastic modulus over long time is more in favour of the latter option, i.e. direct contacts at the origin of thixotropy, as suggested for lime and cement pastes [42-43].

The most striking observation concerns the rheological behaviour of these suspensions in the solid regime: the significant increase of the elastic modulus (for small stress amplitude oscillations) as a function of the stress applied during creep test. This contrasts with observations for other simple or thixotropic yield stress fluids of various structures (clay suspension, gel, emulsion) for which this elastic modulus remains remarkably constant up to a stress equal to the yield stress [52]. The latter observation was explained by the fact that the structure at the origin of the solid regime, even it is deformed and if some plastic deformation takes place inside it, is essentially maintained and still forms the backbone of the material structure under any conditions in the solid regime. More precisely, the fact that this elastic modulus remains constant means that the characteristics of the interactions between the elements of the materials have not significantly changed during the deformation, even if significant local deformation of the contact between neighbouring elements occurred. 
The situation is apparently different for the water-CB suspensions: the deformation of the structure induces some change, i.e. a stiffening of the structure. Some similar stiffening before yielding has been reported experimentally in some cases [51]-[57-58] for soft gels which are quite different systems since they do not flow after yielding but fail like solids. It was also observed in numerical simulations of dilute model colloidal gels [50], [59], suggesting a global scenario in which the topology is essentially responsible of the non-linear mechanical response of these soft gels rather than the nature of interactions (weak or strong) between particles. Stiffening or strain hardening might then result from the elongation of the structure responsible of stress bearing in the gel [50].

In this context it is important to first discuss the possible role of GA in this behavior. GA molecules embedded between particles may indeed tend to add some elasticity to the medium. More precisely, they may contribute to the total stress tensor. We then can write this tensor as the sum of a term due to these interactions plus a term due to direct interactions between particles. However, with a polymeric system we do not expect thixotropic effects since possible restructuring results from fast thermal agitation. Thus, the very large increase of the elastic modulus of the suspension in time (cf. Figure 6) essentially results from the variations of the second stress component, associated with particle interactions, and the possible elastic modulus resulting from GA is necessarily smaller than the minimum elastic modulus observed (a short time after preshear).

Let us now examine the elastic modulus increase after some deformation in the solid regime (Figure $9 b)$. The fact that the elastic modulus of the suspension still significantly increases in time proportionally to the level it has reached thanks to the strain stiffening effect, definitely means that the physical origin of the strain stiffening and thixotropy is the same, i.e. the contacts between particles, whereas GA elements do not play any role in strain stiffening. The demonstration of this point is as follows. in the context of the above decomposition of the stress tensor, let us assume that GA particles induce an elastic modulus component due to the imposed stress, i.e. $G_{G A}^{\prime}(\tau)$, which is independent of time for the reasons above described, while the term due to direct particles interactions $\left(G_{\tau=0}^{\prime}(t)\right)$ just varies with time (thixotropy). Then the total elastic modulus would write $G_{\tau}(t)=G_{G A}^{\prime}(\tau)+G_{\tau=0}^{\prime}(t)$, which strongly differs from the trends observed (i.e. $G_{\tau}^{\prime}(t)=a(\tau) G_{\tau=0}^{\prime}(t)$ ) for large values of stresses, i.e. when $G_{G A}^{\prime}(\tau)$ becomes significantly larger than $G_{\tau=0}^{\prime}(t)$. In other words, to get the similar time variations (see Figure 9 ) at different applied stress levels, of the elastic modulus, we need the term due to direct interactions to be dominant and proportional to the initial elastic modulus increase due to applied stress.

Here, since we are basically dealing with a structure based on attractive links between particles throughout the system, which is at the origin of the standard thixotropic and yielding properties, we would expect to keep a constant elastic modulus after some deformation in the solid regime, as for other thixotropic yield stress fluids [52]. The fact that this does not occur means that the deformation induces some evolution of either the spatial arrangement or/and the local characteristics of the interaction between the particles. The former process would lead to an increase of the number of links between particles, thanks to the structure deformation in some way similar to the scenario suggested for soft gels [50] (from numerical simulations) and for CB suspensions in oil [13] (from observations of conductivity increase during creep tests). However, it cannot lead to a large increase of the number of links except if the structure is significantly more concentrated, which is not possible as the structure should still span throughout the medium. As a consequence we hardly see how this effect alone could lead to a large increase of the apparent elastic modulus with deformation (up to 10 times the elastic modulus at low deformation) observed with our suspensions. 
We thus suggest an alternative explanation. We assume that deformations in the stiffening regime of $C B$ suspensions are small enough so that the contacts between particles are not broken and the macroscopic structure is not affected by the deformations, i.e. the number of links between the particles remain roughly constant. However, the deformation of the sample induces local relative movements of particles in contact, which can then explore and fall in more jammed positions, which would induce the stiffening of the material. In this context the peculiar shape of elementary particles of aqueous carbon black might play some significant role in this effect. Indeed, these particles (the smallest units) are not spherical as they result from irreversible aggregation of particles, and they exhibit a large roughness (of the order of the elementary particle size, i.e. $30 \mathrm{~nm}$ ). The initial contact, i.e. the relative position of two neighbouring particles, was formed during the material preparation as these particles met at random. The slight relative rotation of particles in contact due to deformation would then force them to explore various other configurations of contact between the two rough surfaces, so that they could progressively develop stronger interactions. In such a process the particle contacts becoming stiffer would be less deformed in the next step of macroscopic deformation, so that they would not explore significantly different configuration, while the other contact could pursue their exploration and finally reach a stiffer contact configuration, and so on.

The validity of this scheme may be tested from our experimental data. Indeed, in that case we can expect that the increase of strength of the link, and thus the increase of the elastic modulus, is proportional to the local relative motion (with some rotation) of the particles, which is proportional to the deformation. Under these conditions the relative increase of elastic modulus should be a function of the deformation only, i.e. $\left[G_{\tau}^{\prime}-G_{\tau=0}^{\prime}(\phi)\right] / G_{\tau=0}^{\prime}(\phi)=a(\tau)-1=f(\gamma(\tau))$. In order to check that we look at the evolution of the factor $a(\tau)-1$ as a function of the deformation of the material for different concentrations (see Figure 12). We see that all the data approximately fall along a master curve of slope 1 , meaning that whatever the volume fraction and ionic strength, the relative increase of elastic modulus can be expressed at first order as $a(\tau)-1 \approx k \gamma$, with $k \approx 6$ independent of the concentration. Note that a significant scattering is observed at very low deformation (typically below $10 \%$ ), which is expected considering that in this range of deformation $a(\tau)$ is close to 1 so that $a(\tau)-$ 1 is negligible.

The same reasoning should apply to suspensions with various ionic forces, and effectively we observe that $a(\tau)-1$ in that case follows the same master curve (see Figure 12), which confirms the consistency of our assumption. Finally, the (approximate) linearity of the function $f$ with $\gamma$ suggests that the physical origin of the increase of the interaction level between particles is essentially geometrical. 


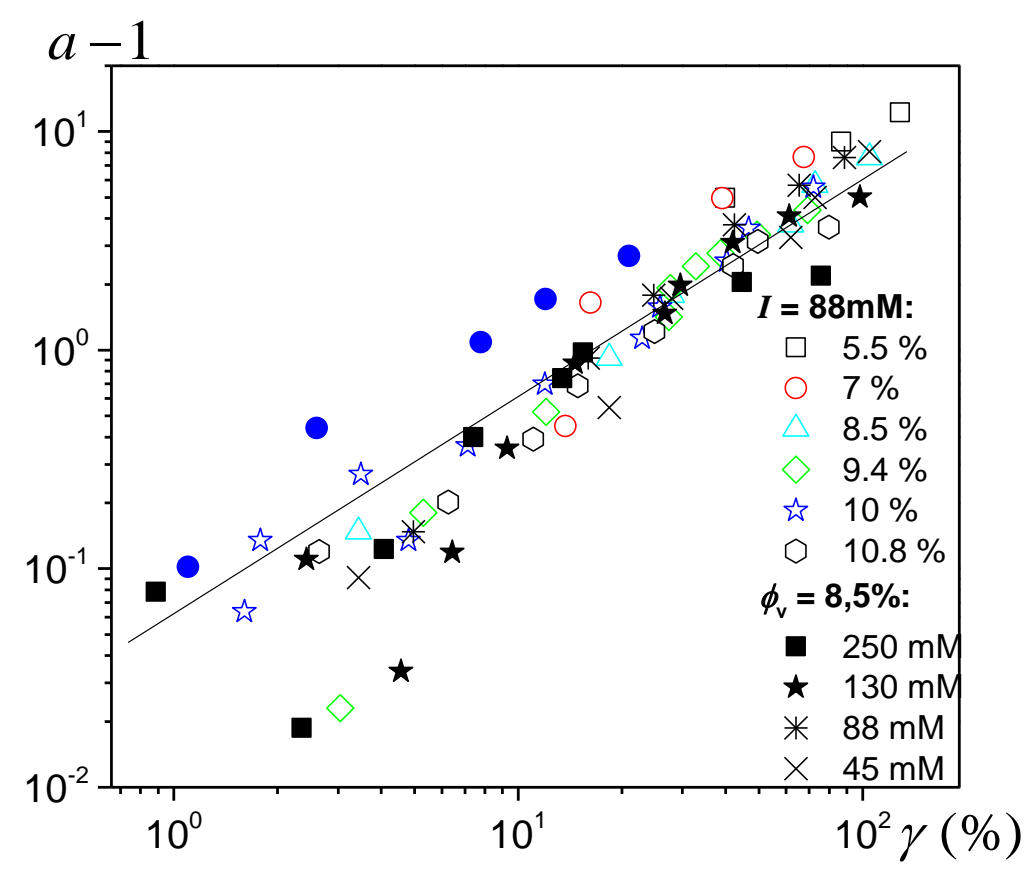

Figure 12. Relative increase of the elastic modulus $(a-1)$ resulting from creep tests as a function of the induced deformation, for the water $C B$ suspensions at different concentrations of particles and different ionic strengths. The line has a slope 1 . The blue filled circles correspond to similar measurements for one material $\left(\Phi_{\mathrm{v}}=8.5 \%\right.$ and $I=$ $88 \mathrm{mM}$ ) carried out after stress release at the end of creep tests.

A further proof of the straightforward dependence of the increase of the elastic modulus for low deformation on the material deformation is provided by imposing another history of deformation. Now, we release the stress after each of the creep tests shown in Figure 12, so that the deformation is now back to a residual (plastic) value as explained above, and we start again following in time the elastic modulus at very low deformation amplitude. We again observe an increase of $G^{\prime}$ in time, at a level increasing with the stress level of the previous creep test (see Appendix 5, Figure 18). Actually, we find that the coefficient $a-1$ also increases linearly with the current (residual) deformation of the material, reached after stress release (see Figure 12).

These results not only show the dependence of the strain stiffening on the sample deformation but also the partial recovery of the process when the deformation is decreased.

\section{Conclusion}

We have studied experimentally the complete (liquid and solid regimes) rheological behaviour of $C B$ suspensions. Differently from most previous rheological studies, we used water as suspending media and gum Arabic to bypass CB particle hydrophobicity. Unlike oil suspensions, aqueous CB suspensions exhibit a "standard" thixotropic yield stress behaviour. Their yield stress and elastic modulus significantly increase with time at rest, but these effects are erased by shear. Furthermore, the solid to liquid transition is abrupt with a discontinuity of the shear rate associated with a viscosity bifurcation effect. 
However, our investigation of their solid regime reveals a striking stiffening effect, i.e. increase of the elastic modulus with deformation amplitude, unlike most simple or thixotropic yield stress fluids. This effect was then better characterized from measurements of the elastic modulus for low strain amplitude in various (larger) deformation states of the material in the solid regime. This elastic modulus appears to increase by a factor up to about 10 as the applied stress approaches the yield stress.

Such an effect resembles that observed for soft gels [51]-[57-58] when measuring the elastic modulus for increasing strain amplitude. There are however several significant differences: in our case the material is and remains a fluid, as a strong shear at any time during the process can fully rejuvenate it and we can start again a test which will lead to the same results; in contrast, soft gels fracture beyond a critical deformation when the strain stiffening effect is maximum. The strain stiffening for $C B$ suspensions appears to be consistent with an explanation in terms of evolution of the individual contacts between particles as a result of relative rotation due to local deformation. This scheme is supported by the demonstration that the strain stiffening is a simple increasing function of the deformation, whatever the history of this deformation (i.e. associated with various stress histories), which also means that the effect is reversible, in contrast with one observation [57] with a soft gel. Finally these results show that the elastic modulus of $C B$ suspensions can be tuned to any value, thanks to an appropriate stress history.

CB suspensions play a key role in different redox flow battery where it is used as liquid electrode [8] potentially enhancing the conductivity in the percolation regime. Future possible works could attempt to see whether these original strain stiffening effect, if effectively associated with a "better" contact between particles, is at the origin of conductivity increase.

Acknowledgements: We acknowledge the support of the French National Research Agency within the frame of the grant ANR-17-CE05-0023-04.

\section{Appendix 1: Interaction potential}

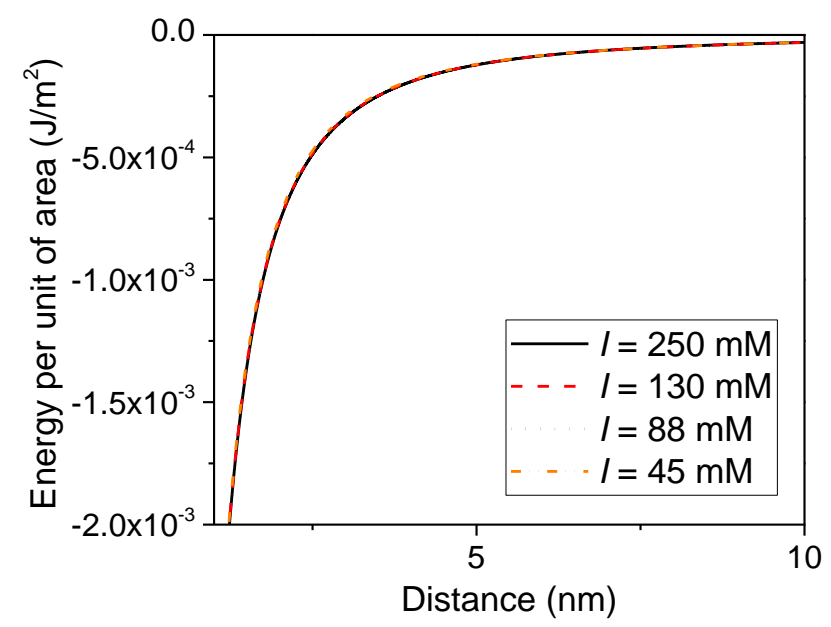

Figure 13: Total interaction potential according to equation (1) for different values of the ionic strength. 
Appendix 2: Strain stiffening in strain sweep dynamic tests

The stiffening before yielding seems to decrease with the ionic strength (see Figure 14). However, classical dynamic tests (ramp of strain amplitude) tend to fail at detecting stiffening at high value of ionic strength where superimposition tests show unambiguously that there is still stiffening. Also, such tests tend to fail at detecting stiffening at high volume fraction (see Figure 15), where again superimposition tests show unambiguously that there is still stiffening.

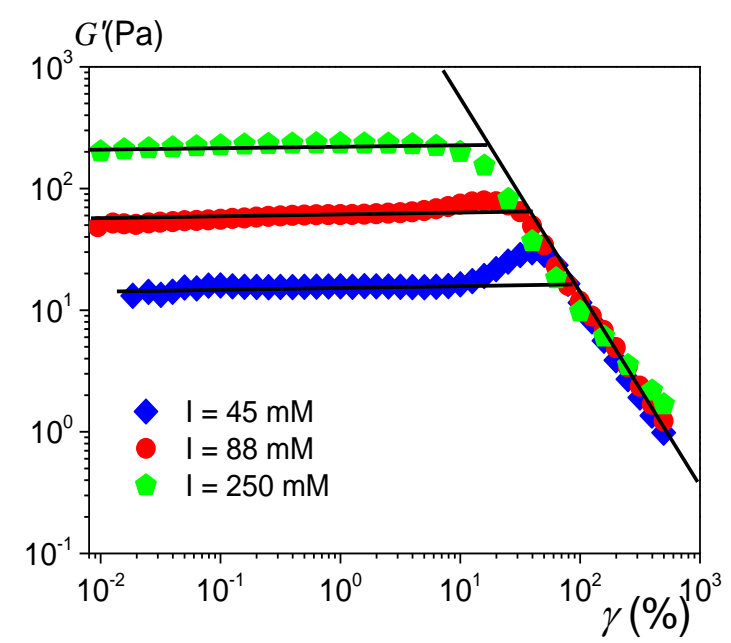

Figure 14: Oscillatory amplitude sweep strain for various ionic strengths and $\Phi_{v}=8.5 \%$.

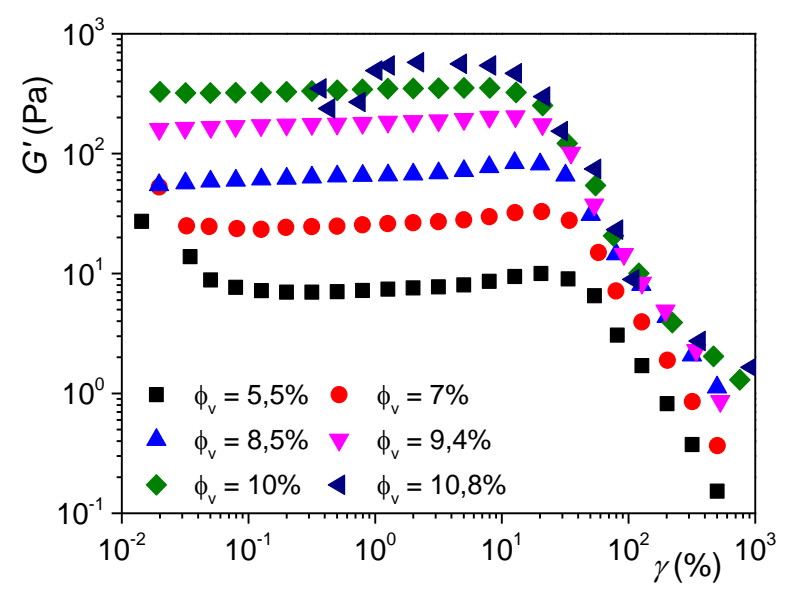

Figure 15. Oscillatory amplitude sweep strain for various volume fractions and $I=$ $88 \mathrm{mM}$.

Appendix 3: Erasing strain stiffening by preshear

Before each value of superimposition tests on Figure 9, the material is first presheared $\left(200 \mathrm{~s}^{-1}\right)$ then left at rest during $60 \mathrm{~s}$ in order to bring back the sample in the same initial state. It appears that during 
the resting time, $G^{\prime}$ always follows the same evolution, similar to that observed before any creep test (see Figure 16). This means that the preshear has erased any effect of strain stiffening observed during the creep tests, and this also proves that our material did not undergo any significant change due to chemical or drying effects during our experiments.

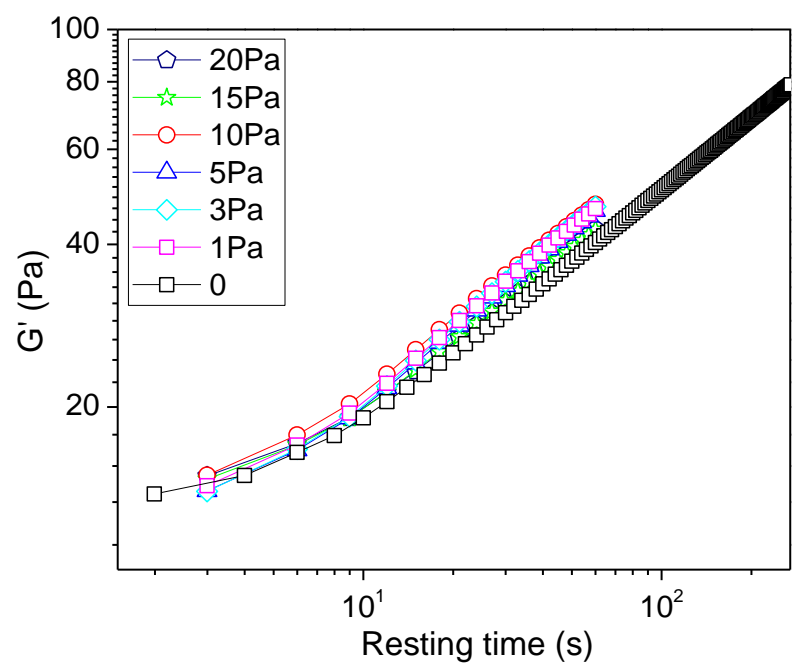

Figure 16: Elastic modulus as a function of resting time measure before each superimposition test of figure 9 .

Appendix 4: Yield stress and elastic modulus as a function of the concentration.

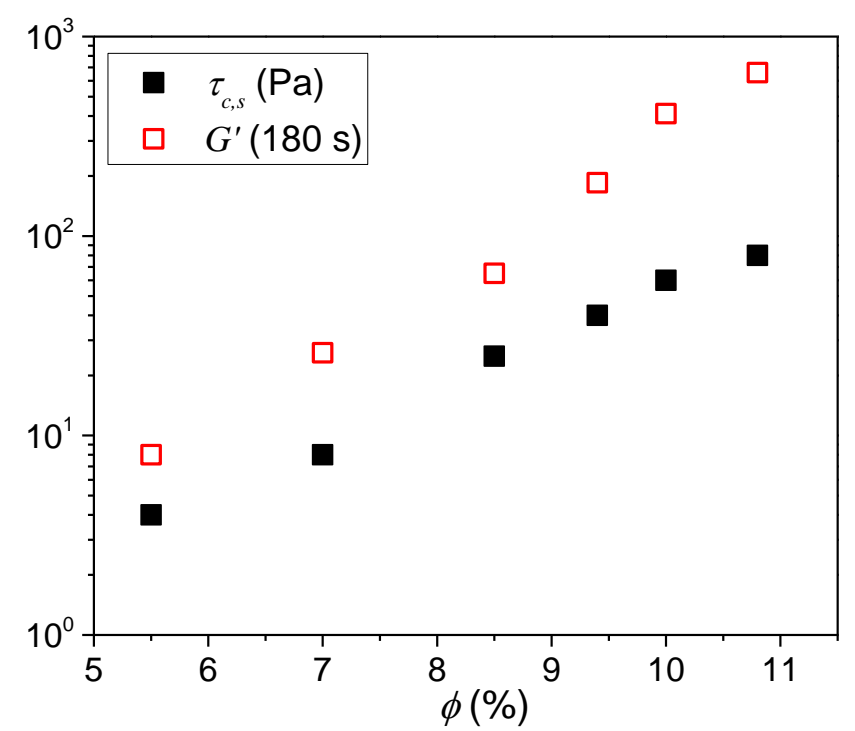

Figure 17: Static yield stress and elastic modulus after respectively $60 \mathrm{~s}$ and $180 \mathrm{~s}$ at rest as a function of concentration.

Appendix 5: Partial recovery of strain stiffening effect after strain recovery 
Here we look at the strain stiffening effect after releasing the stress during which the measure was made initially. We can then compare the new strain stiffening effect, after partial strain recovery (some irreversible strain subsists), to that observed during the previous creep test (see Figure 17).

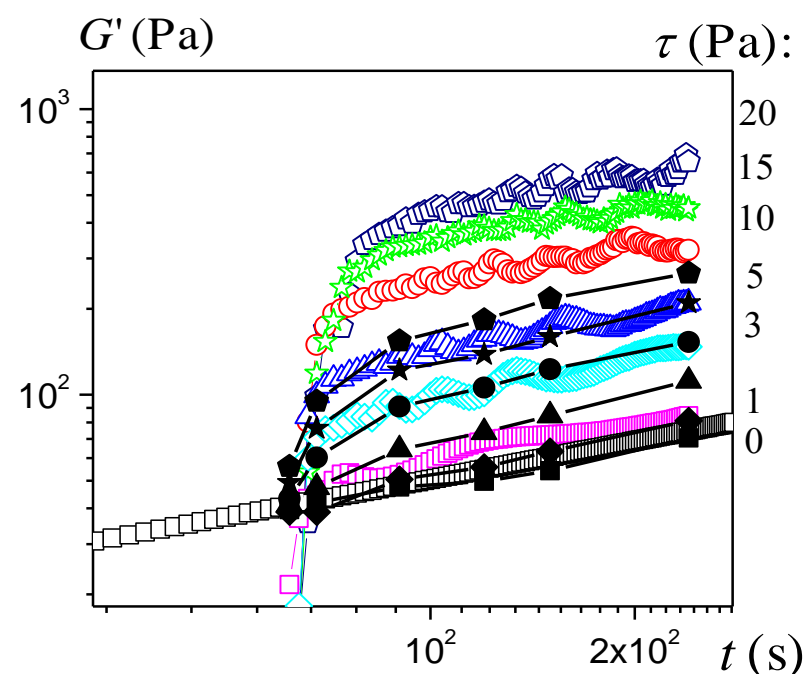

Figure 18: Stiffening of aqueous CB suspension ( $\Phi_{v}=8.5 \%$ and $I=88 \mathrm{mM}$ ) for different deformation history. The open symbols are the measures of $\mathrm{G}^{\prime}$ simultaneously with stress during a superimposed tests. The filled symbols are the measurement of $\mathrm{G}^{\prime}$ after releasing the stress corresponding to the respective open symbols.

\section{References}

[1] B. Omnès, S. Thuillier, P. Pilvin, Y. Grohens, and S. Gillet, "Effective properties of carbon black filled natural rubber: Experiments and modeling," Compos. Part A Appl. Sci. Manuf., vol. 39, no. 7, pp. 1141-1149, 2008, doi: https://doi.org/10.1016/j.compositesa.2008.04.003.

[2] A. Robisson, "A simple analogy between carbon black reinforced rubbers and random threedimensional open-cell solids," Mech. Mater., vol. 42, no. 11, pp. 974-980, 2010, doi: https://doi.org/10.1016/j.mechmat.2010.08.001.

[3] E. Yousif and R. Haddad, "Photodegradation and photostabilization of polymers, especially polystyrene: review," Springerplus, vol. 2, no. 1, p. 398, 2013, doi: 10.1186/2193-1801-2-398.

[4] K. Yamaguchi, I. Sasaki, I. Nishizaki, S. Meiarashi, and A. Moriyoshi, "Effects of Film Thickness, Wavelength, and Carbon Black on Photodegradation of Asphalt," J. Japan Pet. Inst., vol. 48, no. 3, pp. 150-155, 2005, doi: 10.1627/jpi.48.150.

[5] R. Spahr, M. E. and Rothon, "Carbon Black as a Polymer Filler," in Fillers for Polymer Applications, Springer., 2017, pp. 261-291.

[6] K. Dai, X.-B. Xu, and Z.-M. Li, “Electrically conductive carbon black (CB) filled in situ microfibrillar poly(ethylene terephthalate) (PET)/polyethylene (PE) composite with a selective CB distribution," Polymer (Guildf)., vol. 48, no. 3, pp. 849-859, 2007, doi: https://doi.org/10.1016/j.polymer.2006.12.026.

[7] M. Youssry, L. Madec, P. Soudan, M. Cerbelaud, D. Guyomard, and B. Lestriez, "Non-aqueous carbon black suspensions for lithium-based redox flow batteries: Rheology and simultaneous rheo-electrical behavior," Phys. Chem. Chem. Phys., vol. 15, no. 34, pp. 14476-14486, 2013, doi: $10.1039 / \mathrm{c} 3 \mathrm{cp} 51371 \mathrm{~h}$. 
[8] H. Parant, G. Muller, T. Le Mercier, J. M. Tarascon, P. Poulin, and A. Colin, "Flowing suspensions of carbon black with high electronic conductivity for flow applications: Comparison between carbons black and exhibition of specific aggregation of carbon particles," Carbon N. Y., vol. 119, pp. 10-20, 2017, doi: 10.1016/j.carbon.2017.04.014.

[9] C. O. Osuji, C. Kim, and D. A. Weitz, "Shear thickening and scaling of the elastic modulus in a fractal colloidal system with attractive interactions," Phys. Rev. E - Stat. Nonlinear, Soft Matter Phys., vol. 77, no. 6, pp. 8-11, 2008, doi: 10.1103/PhysRevE.77.060402.

[10] C. Perge, N. Taberlet, T. Gibaud, and S. Manneville, "Time dependence in large amplitude oscillatory shear: A rheo-ultrasonic study of fatigue dynamics in a colloidal gel," J. Rheol. (N. Y. N. Y)., vol. 58, no. 5, pp. 1331-1357, 2014, doi: 10.1122/1.4887081.

[11] T. Gibaud, D. Frelat, and S. Manneville, "Heterogeneous yielding dynamics in a colloidal gel," Soft Matter, vol. 6, no. 15, pp. 3482-3488, 2010, doi: 10.1039/c000886a.

[12] T. Gibaud, C. Perge, S. B. Lindström, N. Taberlet, and S. Manneville, "Multiple yielding processes in a colloidal gel under large amplitude oscillatory stress," Soft Matter, vol. 12, no. 6, pp. 1701-1712, 2016, doi: 10.1039/C5SM02587G.

[13] A. Helal, T. Divoux, and G. H. McKinley, "Simultaneous Rheoelectric Measurements of Strongly Conductive Complex Fluids," Phys. Rev. Appl., vol. 6, no. 6, pp. 1-19, 2016, doi: 10.1103/PhysRevApplied.6.064004.

[14] C. O. Osuji and D. A. Weitz, "Highly anisotropic vorticity aligned structures in a shear thickening attractive colloidal system," Soft Matter, vol. 4, no. 7, pp. 1388-1392, 2008, doi: 10.1039/b716324j.

[15] V. Grenard, N. Taberlet, and S. Manneville, "Shear-induced structuration of confined carbon black gels: Steady-state features of vorticity-aligned flocs," Soft Matter, vol. 7, no. 8, pp. 3920-3928, 2011, doi: 10.1039/c0sm01515f.

[16] F. Pignon, A. Magnin, and J. M. Piau, "Butterfly light scattering pattern and rheology of a sheared thixotropic clay gel," Phys. Rev. Lett., vol. 79, no. 23, pp. 4689-4692, 1997, doi: 10.1103/PhysRevLett.79.4689.

[17] Z. Varga et al., "Hydrodynamics control shear-induced pattern formation in attractive suspensions," Proc. Natl. Acad. Sci. U. S. A., vol. 116, no. 25, pp. 12193-12198, 2019, doi: 10.1073/pnas.1901370116.

[18] G. Ovarlez, L. Tocquer, F. Bertrand, and P. Coussot, "Rheopexy and tunable yield stress of carbon black suspensions," Soft Matter, vol. 9, no. 23, pp. 5540-5549, 2013, doi: 10.1039/c3sm27650c.

[19] A. Narayanan, F. Mugele, and M. H. G. Duits, "Mechanical History Dependence in Carbon Black Suspensions for Flow Batteries: A Rheo-Impedance Study," Langmuir, vol. 33, no. 7, pp. 1629-1638, 2017, doi: 10.1021/acs.langmuir.6b04322.

[20] J.B. Hipp, J.J. Richards, N.J. Wagner, "Structure-property relationships of sheared carbon black suspensions determined by simultaneous rheological and neutron scattering measurements", 63, 423, https://doi.org/10.1122/1.5071470

[21] A. S. Negi and C. O. Osuji, "Dynamics of internal stresses and scaling of strain recovery in an aging colloidal gel," Phys. Rev. E - Stat. Nonlinear, Soft Matter Phys., vol. 80, no. 1, pp. 1-4, 2009, doi: 10.1103/PhysRevE.80.010404.

[22] J. Sprakel, S.N. Lindström, T.E. Kodger, D.A. Weitz, Stress enhancement in the delayed yielding 
of colloidal gels, Phys. Rev. Lett., 106, 248303 (2011)

[23] Z. Li et al., "Aqueous semi-solid flow cell: demonstration and analysis," Phys.Chem.Chem.Phys., vol. 2, pp. 15833-15839, 2013, doi: 10.1039/c3cp53428f.

[24] V. Trappe and D. A. Weitz, "Scaling of the viscoelasticity of weakly attractive particles," Phys. Rev. Lett., vol. 85, no. 2, pp. 449-452, 2000, doi: 10.1103/PhysRevLett.85.449.

[25] Y. Y. Won, S. P. Meeker, V. Trappe, D. A. Weitz, N. Z. Diggs, and J. I. Emert, "Effect of temperature on carbon-black agglomeration in hydrocarbon liquid with adsorbed dispersant," Langmuir, vol. 21, no. 3, pp. 924-932, 2005, doi: 10.1021/la047906t.

[26] W. Benton, “EBritannica," in Encyclopedia Britannica, Chicago, 1966, pp. 257-259.

[27] Y. Zhang, A. Narayanan, F. Mugele, M. A. Cohen Stuart, and M. H. G. Duits, "Charge inversion and colloidal stability of carbon black in battery electrolyte solutions," Colloids Surfaces $A$ Physicochem. Eng. Asp., vol. 489, pp. 461-468, 2016, doi: 10.1016/j.colsurfa.2015.08.041.

[28] H. P. Boehm, "Surface oxides on carbon and their analysis: a critical assessment," Carbon $N$. Y., vol. 40, no. 2, pp. 145-149, 2002, doi: 10.1016/S0008-6223(01)00165-8.

[29] H. Ridaoui, A. Jada, L. Vidal, and J. B. Donnet, "Effect of cationic surfactant and block copolymer on carbon black particle surface charge and size," Colloids Surfaces A Physicochem. Eng. Asp., vol. 278, no. 1-3, pp. 149-159, 2006, doi: 10.1016/j.colsurfa.2005.12.013.

[30] M. V. Smoluchowski, "Handbook of Electricity and Magnetism," Leipzig, 1921.

[31] J. Israelachvili, Intermolecular and Surface Forces. 2011.

[32] S. B. Johnson, A. S. Russell, and P. J. Scales, "Volume fraction effects in shear rheology and electroacoustic studies of concentrated alumina and kaolin suspensions," Colloids Surfaces $A$ Physicochem. Eng. Asp., vol. 141, no. 1, pp. 119-130, 1998, doi: 10.1016/S09277757(98)00208-8.

[33] Y. Matsumura, C. Satake, M. Egami, and T. Mori, "Interaction of gum arabic, maltodextrin and pullulan with lipids in emulsions," Biosci. Biotechnol. Biochem., vol. 64, no. 9, pp. 1827-1835, 2000, doi: 10.1271/bbb.64.1827.

[34] H. Parant, "Le concept d'électrodes liquides de carbone appliqué au domaine des batteries en flux : étude et application aux matériaux d'intercalation du lithium," Université de Bordeaux, 2017.

[35] M. L. Jayme, D. E. Dunstan, and M. L. Gee, "Zeta potentials of gum arabic stabilised oil in water emulsions," Food Hydrocoll., vol. 13, no. 6, pp. 459-465, 1999, doi: 10.1016/S0268005X(99)00029-6.

[36] P. T. Callaghan, Principles of Nuclear Magnetic Resonance Microscopy. Clarendon Press, 1991.

[37] J. S. Raynaud, P. Moucheront, J. C. Baudez, F. Bertrand, J. P. Guilbaud, and P. Coussot, "Direct determination by nuclear magnetic resonance of the thixotropic and yielding behavior of suspensions," J. Rheol. (N. Y. N. Y)., vol. 46, no. 3, pp. 709-732, 2002, doi: 10.1122/1.1463420.

[38] P. Coussot, Rheometry of pastes, suspensions, and granular materials: applications in industry and environment, Jhon Wiley. 2005.

[39] P. Coussot, Q. D. Nguyen, H. T. Huynh, and D. Bonn, "Viscosity bifurcation in thixotropic, yielding fluids," J. Rheol. (N. Y. N. Y)., vol. 46, no. 3, pp. 573-589, 2002, doi: 10.1122/1.1459447. 
[40] P. Coussot et al., "Coexistence of Liquid and Solid Phases in Flowing Soft-Glassy Materials," Phys. Rev. Lett., vol. 88, no. 21, p. 218301, May 2002, doi: 10.1103/PhysRevLett.88.218301.

[41] G. Ovarlez, S. Rodts, X. Chateau, and P. Coussot, "Phenomenology and physical origin of shear localization and shear banding in complex fluids," Rheol. Acta, vol. 48, no. 8, pp. 831-844, 2009, doi: 10.1007/s00397-008-0344-6.

[42] M. Fourmentin, G. Ovarlez, P. Faure, U. Peter, D. Lesueur, P. Coussot, "Rheology of lime paste - A comparison with cement paste", Rheologica Acta, 54, 647-654 (2015)

[43] N. Roussel, G. Ovarlez, S. Garrault, and C. Brumaud, "The origins of thixotropy of fresh cement pastes," Cem. Concr. Res., vol. 42, no. 1, pp. 148-157, 2012, doi: 10.1016/j.cemconres.2011.09.004.

[44] D. E. V Andrade and P. Coussot, "Brittle solid collapse to simple liquid for a waxy suspension," Soft Matter, vol. 15, no. 43, pp. 8766-8777, 2019, doi: 10.1039/C9SM01517E.

[45] V. Trappe, V. Prasad, L. Cipelletti, P. N. Segre, and D. A. Weitz, "Jamming phase diagram for attractive particles," Nature, vol. 411, no. June, pp. 0-3, 2001, doi: 10.1038/35081021.

[46] F. Bonacci, X. Chateau, E. M. Furst, J. Fusier, J. Goyon, and A. Lemaître, "Contact and macroscopic ageing in colloidal suspensions," Nat. Mater., 2020, doi: 10.1038/s41563-0200624-9.

[47] C. Derec, G. Ducouret, A. Ajdari, and F. Lequeux, "Aging and nonlinear rheology in suspensions of polyethylene oxide--protected silica particles," Phys. Rev. E, vol. 67, no. 6, p. 61403, Jun. 2003, doi: 10.1103/PhysRevE.67.061403.

[48] M. Jaspers, M. Dennison, M. F. J. Mabesoone, F. C. MacKintosh, A. E. Rowan, and P. H. J. Kouwer, "Ultra-responsive soft matter from strain-stiffening hydrogels," Nat. Commun., vol. 5, pp. 1-8, 2014, doi: 10.1038/ncomms6808.

[49] K. Bertula et al., "Strain-Stiffening of Agarose Gels," ACS Macro Lett., vol. 8, pp. 670-675, 2019, doi: 10.1021/acsmacrolett.9b00258.

[50] J. Colombo and E. Del Gado, "Stress localization, stiffening, and yielding in a model colloidal gel," J. Rheol. (N. Y. N. Y)., vol. 58, no. 5, pp. 1089-1116, 2014, doi: 10.1122/1.4882021.

[51] T. Gisler, R. C. Ball, and D. A. Weitz, "Strain hardening of fractal colloidal gels," Phys. Rev. Lett., vol. 82, no. 5, pp. 1064-1067, 1999, doi: 10.1103/PhysRevLett.82.1064.

[52] E. N'Gouamba, J. Goyon, and P. Coussot, "Elastoplastic behavior of yield stress fluids," Phys. Rev. Fluids, vol. 4, no. 12, p. 123301, Dec. 2019, doi: 10.1103/PhysRevFluids.4.123301.

[53] G. Ovarlez and P. Coussot, "Physical age of soft-jammed systems," Phys. Rev. E - Stat. Nonlinear, Soft Matter Phys., vol. 76, no. 1, pp. 1-7, 2007, doi: 10.1103/PhysRevE.76.011406.

[54] G. Ovarlez and X. Chateau, "Influence of shear stress applied during flow stoppage and rest period on the mechanical properties of thixotropic suspensions," Phys. Rev. E - Stat. Nonlinear, Soft Matter Phys., vol. 77, no. 6, pp. 1-14, 2008, doi: 10.1103/PhysRevE.77.061403.

[55] P. Coussot and G. Ovarlez, "Physical origin of shear-banding in jammed systems," vol. 188, pp. 183-188, 2010, doi: 10.1140/epje/i2010-10660-9.

[56] B. J. Park, J. P. Pantina, E. M. Furst, M. Oettel, S. Reynaert, and J. Vermant, “Direct Measurements of the Effects of Salt and Surfactant on Interaction Forces between Colloidal Particles at Water - Oil Interfaces," no. 17, pp. 1686-1694, 2008, doi: 10.1021/la7008804.

[57] G. de Oliveira Reis et al., "Irreversible hardening of a colloidal gel under shear: The smart 
response of natural rubber latex gels," J. Colloid Interface Sci., vol. 539, pp. 287-296, 2019, doi: 10.1016/j.jcis.2018.12.031.

[58] M. Pouzot, T. Nicolai, L. Benyahia, and D. Durand, "Strain hardening and fracture of heat-set fractal globular protein gels," J. Colloid Interface Sci., vol. 293, no. 2, pp. 376-383, 2006, doi: 10.1016/j.jcis.2005.06.074.

[59] M. Bouzid and E. Del Gado, "Network Topology in Soft Gels: Hardening and Softening Materials," Langmuir, vol. 34, no. 3, pp. 773-781, 2018, doi: 10.1021/acs.langmuir.7b02944. 